\title{
EZH2 inhibition sensitizes tamoxifen-resistant breast cancer cells through cell cycle regulation
}

\author{
SI CHEN $^{1 *}$, FAN YAO $^{2 *}$, QINGHUAN XIAO ${ }^{3}$, QIANNAN LIU $^{1}$, YIKUN YANG $^{1}$, \\ XUEJUAN LI $^{1}$, GUANGLIE JIANG ${ }^{1}$, TAKAYOSHI KUNO ${ }^{1,4}$ and YUE FANG ${ }^{1}$
}

\begin{abstract}
${ }^{\mathrm{I}}$ Department of Microbial and Biochemical Pharmacy, School of Pharmacy, China Medical University, Shenyang, Liaoning 110112; ${ }^{2}$ Department of Breast Surgery and Surgical Oncology, Research Unit of General Surgery, The First Affiliated Hospital of China Medical University, Shenyang, Liaoning 110001; ${ }^{3}$ Department of Ion Channel Pharmacology, School of Pharmacy, China Medical University, Shenyang, Liaoning 110112, P.R. China; ${ }^{4}$ Division of Food and Drug Evaluation Science, Kobe University Graduate School of Medicine, Kobe 650-0017, Japan
\end{abstract}

Received July 12, 2017; Accepted November 21, 2017

DOI: $10.3892 / \mathrm{mmr} .2017 .8160$

\begin{abstract}
Enhancer of zeste homologue 2 (EZH2), a catalytic subunit of polycomb repressive complex 2 , is overexpressed in a number of different tumors including breast cancer, and serves important roles in cell cycle regulation, proliferation, apoptosis, tumorigenesis and drug resistance. However, it remains unclear whether EZH2 contributes to tamoxifen resistance in breast cancer. In the present study, the role of $\mathrm{EZH} 2$ in tamoxifen resistance in MCF-7 cells was investigated. EZH2 was overexpressed in MCF-7 tamoxifen-resistant (MCF-7 TamR) cells. EZH2 overexpression decreased the sensitivity of MCF-7 cells to tamoxifen, and EZH2 knockdown improved the sensitivity of MCF-7 TamR cells to tamoxifen. Furthermore, EZH2 knockdown induced cell cycle arrest in MCF-7 TamR cells, accompanied by a decrease in cyclin D1 expression and an increase in p16 expression. EZH2 knockdown reduced p16 gene methylation in MCF-7 TamR cells. These findings suggested that EZH2 overexpression may contribute to tamoxifen resistance in breast cancer, and EZH2 inhibition may reverse tamoxifen resistance in breast cancer by regulating the cell cycle via the demethylation of the p16 gene. Thus, EZH2 inhibitors may be effective for treating tamoxifen resistance in breast cancer.
\end{abstract}

\footnotetext{
Correspondence to: Professor Yue Fang, Department of Microbial and Biochemical Pharmacy, School of Pharmacy, China Medical University, 77 Puhe Road, Shenyang North New Area, Shenyang, Liaoning 110112, P.R. China

E-mail: yfang@cmu.edu.cn

*Contributed equally
}

Key words: enhancer of zeste homolog 2, tamoxifen resistance, cell cycle, breast cancer

\section{Introduction}

Breast cancer is a leading cause of death in women worldwide (1). Tamoxifen, a selective estrogen receptor modulator (SERM), is one of the most effective endocrine therapy for estrogen receptor (ER)-positive breast cancer (2). However, approximately $30-40 \%$ of ER-positive breast cancer patients do not respond to tamoxifen endocrine therapy, and moreover, tumors that initially respond to tamoxifen treatment develop resistance to this drug over time $(3,4)$. The mechanisms underlying tamoxifen resistance are complex and remain unclear, although loss of ER expression or dysregulation of ER co-regulators, and activation of many kinases such as the receptor tyrosine kinase have been found to contribute to tamoxifen resistance (5).

Enhancer of zeste homologue 2 (EZH2), as a histone methyltransferase, is a catalytic subunit of polycomb repressive complex 2 (PRC2) which induces transcriptional inhibition through the tri-methylation of lysine residue 27 on histone $\mathrm{H} 3$ (H3K27m3) (6). Abnormal activities of DNA methyltransferases lead to epigenetic changes of many genes that contribute to carcinogenesis (7). EZH2 plays an important role in many cellular processes such as cell cycle regulation, proliferation, apoptosis, tumorigenesis, and drug resistance (8-10). EZH2 has been found to be overexpressed in a wide range of tumors such as osteosarcoma (11), breast cancer (12), and prostate cancer (13). EZH2 overexpression is associated with poor clinical outcomes $(14,15)$. Inhibition of EZH2 may represent a promising therapeutic strategy for anticancer treatment (16).

Breast cancer is a heterogeneous disease that includes different subtypes defined by ER, progesterone receptor (PR), and human epidermal growth factor receptor 2 (HER2). In addition, Jang et al (17) reported that high EZH2 protein expression was associated with poor survival in patients with Luminal A breast cancer. Moreover, high EZH2 expression has been reported to be associated with unfavorable outcome in the ER-positive breast cancer patients following tamoxifen treatment (18). Therefore, it appears that EZH2 overexpression may contribute to tamoxifen resistance in ER-positive breast cancer. However, the role of EZH2 in tamoxifen resistance has not been investigated yet. 
In the present study, we established a tamoxifen resistant MCF-7 breast cancer cell line, and investigated the role of EZH2 in tamoxifen resistance. The purpose of this study was to explore the mechanisms by which EZH2 mediated tamoxifen resistance in breast cancer cells.

\section{Materials and methods}

Cell culture. The human breast cancer cell line MCF-7 was obtained from the American Type Culture Collection (ATCC, Rockville,MD, USA). MCF-7 cells were cultured in Dulbecco's Modified Eagle Medium (DMEM; Biological Industries, Beit-Haemek, Israel) supplemented with $10 \%$ fetal bovine serum (FBS; Biological Industries), $100 \mathrm{IU} / \mathrm{ml}$ penicillin, and $100 \mathrm{IU} / \mathrm{ml}$ streptomycin (Beyotime, Shanghai, China).

MCF-7 tamoxifen resistant (MCF-7 TamR) cells were selected from MCF-7 parental cells after treatment with 4-hydroxytamoxifen (4-OH TAM; Sigma-Aldrich; Merck KGaA, Darmstadt, Germany) for 10 months as previously reported (19) with some modifications. Briefly, MCF-7 cells were cultured in the phenol red-free RPMI 1640 medium (Gibco; Thermo Fisher Scientific, Inc., Waltham, MA, USA). Cells were treated with $1 \mu \mathrm{M}$ 4-OH TAM for 21 days, followed by incubation with TAM-free medium for 7 days. Survived cells were diluted to obtain the monoclonal cells, which were further cultured in $1 \mu \mathrm{M} 4-\mathrm{OH}$ TAM for 10 months. MCF-7 parental cells grown in the RPMI 1640 medium with $0.1 \%$ ethanol (vehicle) for 10 months were used as control cells. All cultures were maintained in a $5 \% \mathrm{CO}_{2}, 37^{\circ} \mathrm{C}$, and $95 \%$ humidity cell culture incubator.

Cell viability assays. Cell viability was assessed using the 3-(4, 5-dimethylthiazol-2-yl)-2, 5-diphenyl tetrazolium bromide (MTT) assay. Briefly, cells were plated into 96-well plates at a density of 500 cells/well. Cells were cultured for $24 \mathrm{~h}$, and then were treated with different concentrations of 4-OH TAM (0-18 $\mu \mathrm{M})$ for 2 days. MTT solution (20 $\mu$; Solarbio, Beijing, China) was added to each well at a final concentration of $0.05 \mathrm{mg} / \mathrm{l}$ and incubated for $4 \mathrm{~h}$. After MTT solution was removed, $150 \mu 1$ DMSO was added to each well, and mixed carefully. The plate was read at a wavelength of $570 \mathrm{~nm}$ in a microplate reader (Bio-Rad Laboratories, Inc., Hercules, CA, USA). All experiments were performed in triplicated and repeated at least three times.

RNA isolation and RT-qPCR assays. Total RNA was isolated from cells using Trizol reagent (Invitrogen; Thermo Fisher Scientific, Inc., Waltham, MA, USA). Complementary DNA was synthesized with $1 \mu \mathrm{g}$ of total RNA in a $10 \mu \mathrm{l}$ of a reaction mixture (Promega Corporation, Madison, WI, USA) according to the manufacturer's instruction. Quantitative RT-PCR was performed using SYBR Green real-time qPCR kit (Toyobo Life Science, Osaka, Japan) in the Agilent Technologies Stratagene Mx3000P (Agilent Technologies, Inc., Santa Clara, CA, USA). The primer sequences were as follows: 5'-AGGACGGCTCCTCTAACCAT-3' (sense) and 5'-CTTGGTGTTGCACTGTGCTT-3' (antisense) for EZH2; and 5'-TGACGTGGACATCCGCAAAG-3' (sense) and 5'-CTGGAAGGTGGACAGCGAGG-3' (antisense) for $\beta$-actin. The PCR amplification conditions were $10 \mathrm{~min}$ at $95^{\circ} \mathrm{C}$, followed by 40 cycles at $94^{\circ} \mathrm{C}$ for $15 \mathrm{sec}, 60^{\circ} \mathrm{C}$ for $45 \mathrm{sec}$ and $72^{\circ} \mathrm{C}$ for $20 \mathrm{sec}$. Quantitative RT-PCR assays were conducted on a MxPro-Mx3000P (Standalone) Comparative Quantitation (Agilent Technologies, Inc.). All quantitative RT-PCRs were performed in triplicate.

Plasmid construction and transfection. The human genomic cDNA of the EZH2 (NM_004456.4) was amplified by PCR and was subcloned into the XhoI/KpnI site of pcDNA3.1 (+) vector (cat. no. V79020; Invitrogen; Thermo Fisher Scientific, Inc.). The primer sequences were shown in Table I. The construct of the EZH2 expression vector was confirmed by DNA sequencing. MCF-7 cells were transfected with the EZH2 expression vector or control vector using Lipofectamine 2000 Transfection Reagent (Invitrogen; Thermo Fisher Scientific, Inc.) according to the manufacturer's instructions. The transfection efficiency was confirmed by western blot analysis. After transfection for $72 \mathrm{~h}$, cells were used for MTT assay, western blot analysis, and flow cytometry.

siRNA transfection. Cells were seeded into six-well plates at a density of $1.5 \times 10^{5}$ cells/well. Then, siRNAs against EZH2 (target sequence: 5'-CAGACGAGCTGATGAAGTAAA-3'; cat. no. SI00063966; Qiagen GmbH, Hilden, Germany) or negative control siRNAs (target sequence: 5'-AATTCTCCGAACGTG TCACGT-3'; cat. no. 1022076; Qiagen, Hilden, Germany) (20) were transfected into cells using Lipofectamine 2000 Transfection Reagent (Invitrogen; Thermo Fisher Scientific, Inc.) according to the manufacturer's recommendations. The transfection efficiency was confirmed by western blot analysis. After transfection, cells were used for MTT assay, western blot analysis, and flow cytometry.

Western blot analysis. Cells were homogenized in ice-cold RIPA lysis buffer (Beyotime, Shanghai, China) supplemented with $1 \mathrm{mM}$ phenylmethylsulfonyl fluoride (PMSF) (Solarbio) as previously described (21). Protein concentrations were determined using Bicinchoninic Acid Kit (Beyotime). Then, equal amounts of protein $(10-20 \mu \mathrm{g})$ were separated by $12 \%$ SDS-polyacrylamide gel electrophoresis (SDS-PAGE) and electrically transferred to PVDF membranes (GE Healthcare, Chicago, IL, USA). The membranes were blocked with 5\% fat-free milk followed by incubation with primary antibodies against EZH2 (mouse anti-human polyclonal antibody, dilution 1:2,500; cat. no. ab168764; Abcam, Cambridge, MA, USA), cyclin D1 (rabbit anti-human polyclonal antibody, dilution 1:1,000; cat. no. AB32262; Absci, Vancouver, WA, USA), p16 $^{\text {ink4a }}$ (rabbit anti-human polyclonal antibody, dilution 1:1,000; cat. no. 10883-1-AP; Wuhan Sanying Biotechnology, Wuhan, China), ER (rabbit anti-human polyclonal antibody, dilution 1:500; cat. no. 21244-1-AP; Wuhan Sanying Biotechnology), phosphorylated AKT (rabbit anti-human monoclonal antibody, dilution 1:2,000; cat. no. 4060; Cell Signaling Technology, Inc., Danvers, MA, USA), AKT (mouse anti-human monoclonal antibody, dilution 1:2,000; cat. no. 2920; Cell Signaling Technology, Inc.), phosphorylated ERK1/2 (rabbit anti-human monoclonal antibody, dilution 1:2,000; cat. no. 4370; Cell Signaling Technology, Inc.), or ERK1/2 (rabbit anti-human monoclonal antibody, dilution 1:1,000; cat. no. 4695; Cell Signaling Technology, Inc.) at $4^{\circ} \mathrm{C}$ overnight. The membranes 
Table I. Primer sequences used in the present study.

\begin{tabular}{llc}
\hline Oligo name & \multicolumn{1}{c}{ Primer sequence } & Product size (bp) \\
\hline EZH2-F & 5'-TTTAAACTTAAGCTTGGTACCATGGGCCAGACTGGGAAG-3' & 2,256 \\
EZH2-R & 5'-AACGGGCCCTCTAGACTCGAGTCAAGGGATTTCCATTTCTC-3' & 150 \\
p16 MF & 5'-TTATTAGAGGGTGGGGCGGATCGC-3' & \\
p16 MR & 5'-GACCCCGAACCGCGACCGTAA-3' & 151 \\
P16 UF & 5'-TTATTAGAGGGTGGGGTGGATTGT-3' & \\
P16 UR & 5'-CAACCCCAAACCACAACCATAA-3' &
\end{tabular}

EZH2, enhancer of zeste homologue 2; F, forward; R, reverse; MF, forward for methylation analysis; MR, reverse for methylation analysis; UF, forward for unmethylation analysis; UR, reverse for unmethylation analysis.

were then incubated with horseradish peroxidase-linked goat anti-rabbit/mouse secondary antibody (dilution 1:5,000, OriGene Technologies, Inc., Beijing, China) at room temperature for $1.5 \mathrm{~h}$. $\beta$-actin (mouse anti-human monoclonal antibody, dilution 1:1,000; cat. no. TA-09; OriGene Technologies, Inc.) was used as a loading control. Bands were visualized using a chemiluminescence detection system, and was analyzed using the Tanon Gis software (Tanon, China).

Flow cytometry. Cell cycle analyses were performed by flow cytometry. Briefly, after transfection with pcDNA3.1-EZH2 or control pcDNA3.1 as well as siEZH2 or control siRNAs for $72 \mathrm{~h}, \mathrm{MCF}-7$ TamR and parental cells were seeded into six-well plates and treated with 4-OH TAM $(8 \mu \mathrm{M})$ for $48 \mathrm{~h}$. Then, cells were collected, washed, fixed with $75 \%$ ethanol at $-20^{\circ} \mathrm{C}$ for $24 \mathrm{~h}$. Cells stained with the propidium iodide (PI) at a final concentration of $10 \mu \mathrm{l} / \mathrm{ml}$ for $30 \mathrm{~min}$ in the dark. Data acquisitions were performed using a flow cytometer (BD FACSCalibur ${ }^{\mathrm{TM}}$; BD Biosciences, Franklin Lakes, NJ, USA). The percentage of cells in the G1, S, and G2 phases was analyzed. All experiments were repeated at least three times.

Methylation analysis by methylation-specific PCR (MSP). After transfection with pcDNA3.1-EZH2 or control pcDNA3.1 as well as siEZH2 or control siRNAs for $72 \mathrm{~h}$, MCF-7 TamR and parental cells were treated with 4-OH TAM $(8 \mu \mathrm{M})$ for $48 \mathrm{~h}$. Total DNA was extracted from cells using the cell/tissue genomic DNA extraction kit (Beijing Transgen Biotech Co., Ltd., Beijing, China) according to the manufacturer's instructions. DNA $(1 \mu \mathrm{g})$ was bisulfite-treated with the CpGenome ${ }^{\mathrm{TM}}$ DNA modification kit (EMD Millipore, Billerica, MA, USA) according to the manufacturer's protocol. Methylation-specific PCR was carried out to investigate the methylation status of the p16 gene. PCR primers specific to unmethylated and methylated bisulfite-modified DNA (22) are shown in Table I. PCR reactions were performed as follows: $95^{\circ} \mathrm{C}$ for $10 \mathrm{~min}, 94^{\circ} \mathrm{C}$ for $30 \mathrm{sec}$, annealing at $62^{\circ} \mathrm{C}$ for $30 \mathrm{sec}$, and extension at $72^{\circ} \mathrm{C}$ for $30 \mathrm{sec}$; a total of 40 cycles; followed by a final extension at $72^{\circ} \mathrm{C}$ for $10 \mathrm{~min}$. PCR products were separated by $2 \%$ gel electrophoresis, and the density of the methylated band (M) or the unmethylated (U) bands were used to assess the methylation levels of p16. Results from triplicate experiments were used to determine methylation status.

Statistical analysis. Data analyses were performed using the SPSS 16.0 software package (SPSS, Inc., Chicago, IL, USA). Quantitative data are expressed as mean \pm SEM. Student's $\mathrm{t}$ test was used to compare differences among groups. $\mathrm{P}<0.05$ was considered to indicate a statistically significant difference.

\section{Results}

EZH2 is overexpressed in MCF-7 TamR cells. We generated MCF-7 TamR cells by treating MCF-7 parental cells with 4-OH TAM for 10 months. MTT assays showed that in the presence of $2 \mu \mathrm{M} 4-\mathrm{OH}$ TAM, cell viability of both MCF-7 parental and TamR cells was increased in a time-dependent manner, and the growth was faster for MCF-7 TamR cells compared with their parental cells. At 6 days after $2 \mu \mathrm{M}$ 4-OH TAM treatment, cell viability of MCF-7 parental cells was significantly lower compared with MCF-7 TamR cells $(\mathrm{P}<0.05$; Fig. 1A). In addition, in the presence of $4-\mathrm{OH}$ TAM, cell viability of MCF-7 parental cells was decreased in a concentration-dependent manner, and cell viability of MCF-7 TamR cells was significantly higher than that of MCF-7 parental cells at concentrations $\geq 4 \mu \mathrm{M} 4-\mathrm{OH}$ TAM for 2 days $(\mathrm{P}<0.05$; Fig. 1B). These results indicated that MCF-7 TamR cells were resistant to tamoxifen.

We further investigated EZH2 expression in MCF-7 TamR cells. Quantitative RT-PCR results showed that the EZH2 mRNA expression was significantly higher in MCF-7 TamR cells than in MCF-7 parental cells $(\mathrm{P}<0.05$; Fig. 1C). Western blot analysis showed that EZH2 expression was significantly increased in MCF-7 TamR cells compared with MCF-7 parental cells $(\mathrm{P}<0.01$; Fig. 1D). The treatment of $4-\mathrm{OH}$ TAM decreased the EZH2 expression levels in a dose- and time-dependent manner (Fig. 1E and F). We also examined the ER expression levels in MCF-7 TamR cells and their parental cells, and found that there was not significant different from these two cell lines (Fig. 1D).

EZH2 overexpression decreases tamoxifen sensitivity of MCF-7 cells. We then investigated the role of EZH2 in tamoxifen sensitivity by transfecting MCF-7 parental cells with pcDNA3.1-EZH2 expression vectors. Western blot analysis 

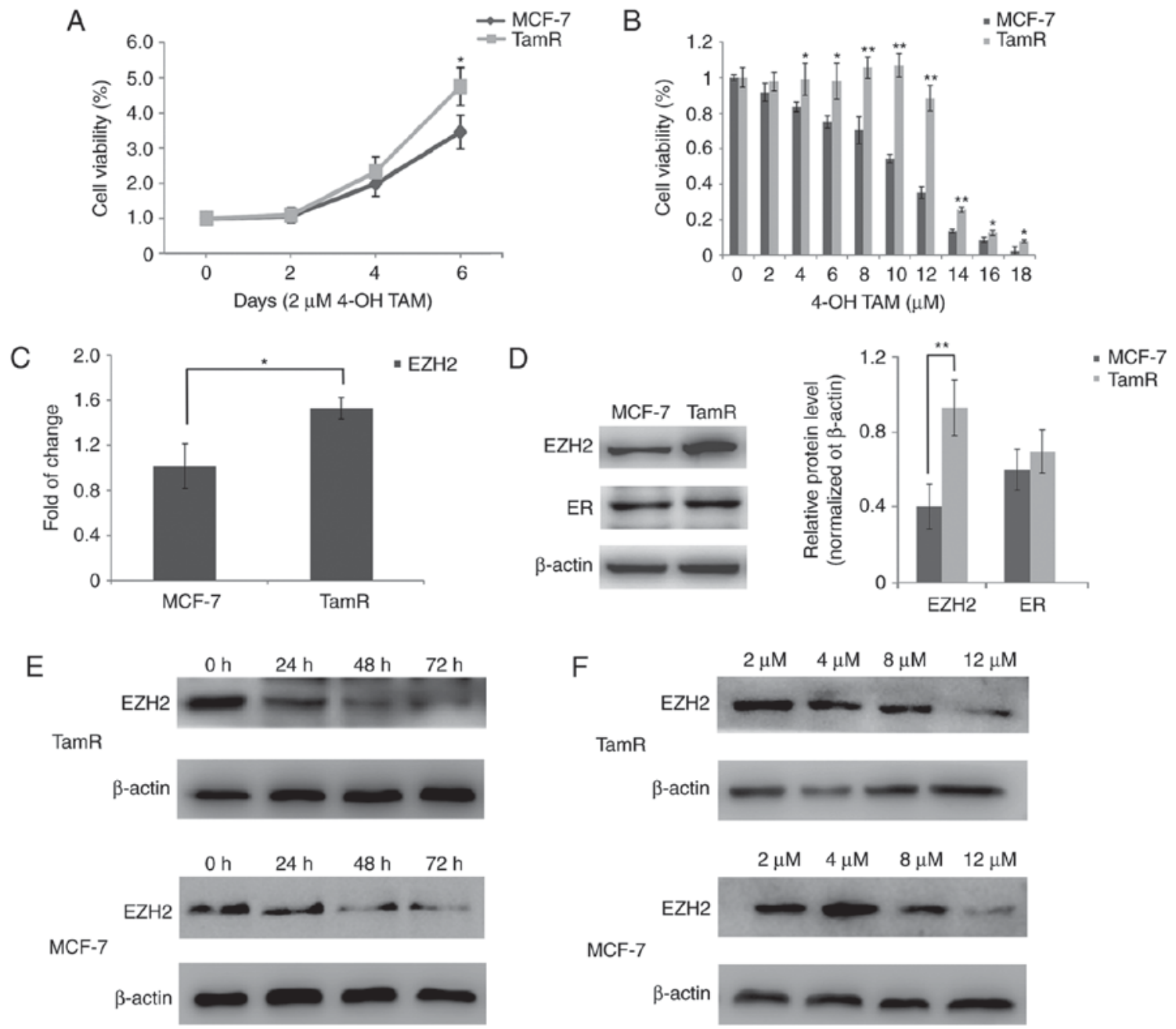

Figure 1. EZH2 was overexpressed in TamR MCF-7 cells. An MTT assay revealed the cell viabilities of MCF-7 TamR and the parental cells in the presence of (A) $2 \mu \mathrm{M}$ 4-OH TAM for 2-6 days $(\mathrm{n}=3)$ and (B) different concentrations of $4-\mathrm{OH}$ TAM $(2-18 \mu \mathrm{M})$ for 2 days $(\mathrm{n}=3)$. ${ }^{*} \mathrm{P}<0.05$ and ${ }^{* * *} \mathrm{P}<0.01$ vs. MCF-7 cells. (C) Reverse transcription-quantitative polymerase chain reaction analysis showed the expression of EZH2 mRNA in MCF-7 TamR and their parental cells. $\beta$-actin was used as a loading control $(\mathrm{n}=3)$. " $\mathrm{P}<0.05$, as indicated. (D) Western blot analysis revealed the protein expression of EZH2 or ER in MCF-7 TamR and their parental cells. $\beta$-actin was used as a loading control. ${ }^{* *} \mathrm{P}<0.01$, as indicated. Western blotting assay showed the protein expression of EZH2 in MCF-7 TamR and parental cells in the presence of (E) $12 \mu \mathrm{M}$ 4-OH TAM for 0-3 days and (F) different concentrations of 4-OH TAM (0-12 $\mu \mathrm{M})$ for 2 days. $\beta$-actin was used as a loading control. EZH2, enhancer of zeste homologue 2; 4-OH TAM, 4-hydroxytamoxifen; ER, estrogen receptor; TamR, tamoxifen resistant.

showed that EZH2 expression was significantly increased in MCF-7 parental cells transfected with pcDNA3.1-EZH2 vectors (Fig. 2A). MTT assays showed that cell viability of EZH2-overexpressing MCF-7 cells was significantly increased in the presence of $4-\mathrm{OH}$ TAM $(\geq 4 \mu \mathrm{M})$ compared with control cells $(\mathrm{P}<0.05$; Fig. 2B). In addition, cell viability of EZH2-overexpressing MCF-7 cells was significantly increased after $8 \mu \mathrm{M}$ 4-OH TAM treatment for 48-72 $\mathrm{h}$ compared with control cells $(\mathrm{P}<0.01 ;$ Fig. $2 \mathrm{C})$. These results suggested that EZH2 overexpression decreased tamoxifen sensitivity of MCF-7 cells.

EZH2 knockdown increases tamoxifen sensitivity of MCF-7 TamR cells. We further investigated whether EZH2 inhibition increased the sensitivity of MCF-7 TamR cells to tamoxifen, using siRNAs against EZH2. Western blot analysis showed that EZH2 expression was decreased in MCF-7 TamR cells transfected with EZH2-siRNAs (Fig. 2D). MTT assays showed that cell viability of MCF-7 TamR cells treated with EZH2-siRNAs was significantly decreased in the presence of
4-OH TAM compared with control cells ( $\mathrm{P}<0.05 ;$ Fig. 2E). Cell viability of MCF-7 TamR cells treated with EZH2-siRNAs was significantly decreased after $8 \mu \mathrm{M} 4-\mathrm{OH}$ TAM treatment for $72 \mathrm{~h}$ compared with control cells $(\mathrm{P}<0.01$; Fig. $2 \mathrm{~F})$. These findings suggested that EZH2 knockdown increased the sensitivity of MCF-7 TamR cells to 4-OH TAM.

EZH2 knockdown induces cell cycle arrest in MCF-7 TamR cells. To understand the role of EZH2 in cell growth of MCF-7 TamR cells, we performed cell-cycle analysis in MCF-7 TamR cells treated with EZH2-siRNAs. Flow cytometry results showed that the percentage of cells in the G0/G1 phase was significantly increased and the percentage of cells in the $\mathrm{S}$ phase was significantly decreased in MCF-7 TamR cells treated with EZH2-siRNAs compared with control cells $(\mathrm{P}<0.05$; Fig. $3 \mathrm{~A}$ and $\mathrm{B})$, suggesting that EZH2 knockdown induced cell cycle arrest in MCF-7 TamR cells.

We then examined the role of EZH2 in cell cycle of MCF-7 parental cells by overexpressing pcDNA3.1-EZH2 vectors. Flow cytometry results showed that the percentage 
A

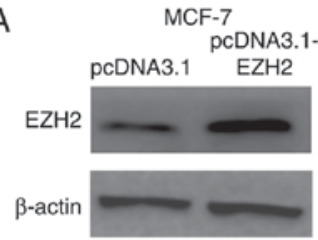

D

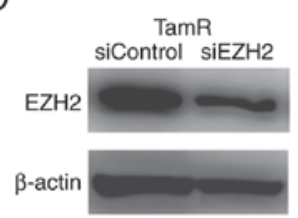

B

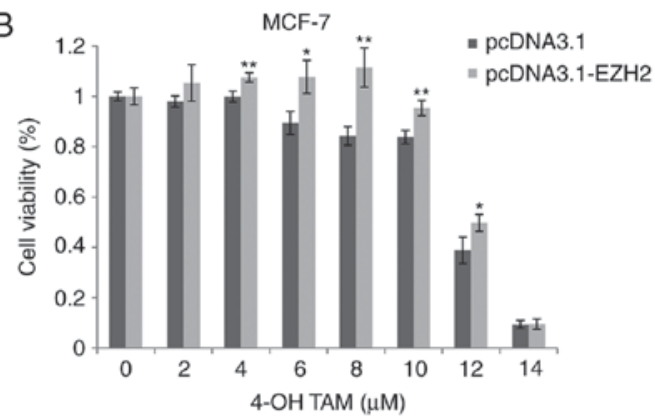

C

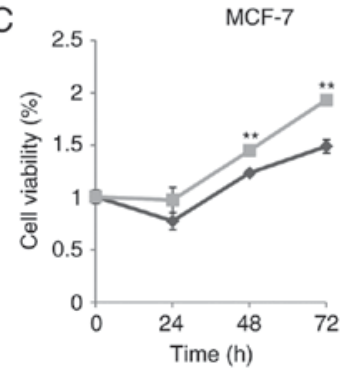

E

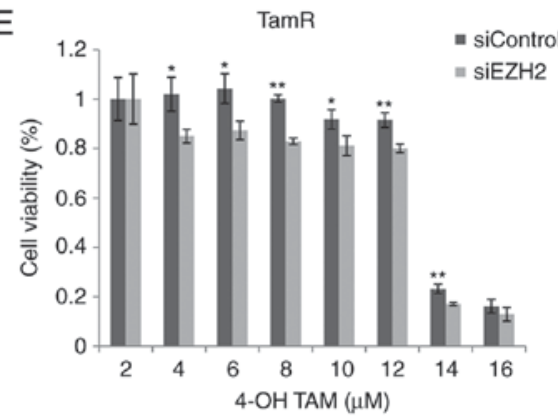

$\mathrm{F}$

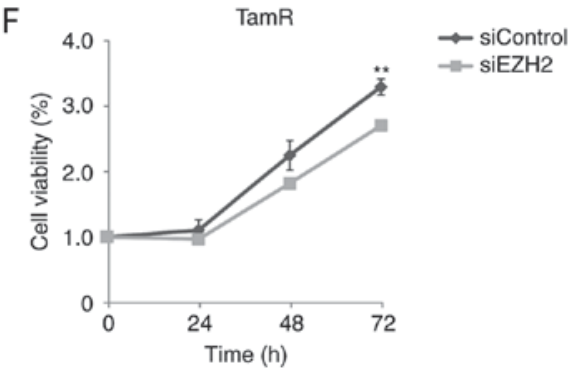

Figure 2. Role of EZH2 in tamoxifen sensitivity. (A) Representative western blot analysis of the protein expression of EZH2 in MCF-7 cells transfected with pcDNA3.1-EZH2 expression vectors or pcDNA3.1 empty vectors for $72 \mathrm{~h}$. $\beta$-actin was used as a loading control. (B) MTT assay revealed the cell viability of MCF-7 cells transfected with pcDNA3.1-EZH2 expression vector or pcDNA3.1 vector for $72 \mathrm{~h}$. Cells were treated with different concentrations of 4-OH TAM $(0-14 \mu \mathrm{M})$ for $48 \mathrm{~h}(\mathrm{n}=3)$. (C) MTT assay showed the cell viability of MCF-7 cells transfected with pcDNA3.1-EZH2 expression vector or pcDNA3.1 vector for $72 \mathrm{~h}$. Cells were treated with 4-OH TAM $(8 \mu \mathrm{M})$ for $0-72 \mathrm{~h}(\mathrm{n}=3)$. ${ }^{*} \mathrm{P}<0.05$ and ${ }^{* *} \mathrm{P}<0.01$ vs. pcDNA3.1 vector. (D) Representative western blot analysis of the protein expression of EZH2 in MCF-7 TamR cells transfected with siRNAs against EZH2 or control siRNAs for 72 h. $\beta$-actin was used as a loading control. (E) MTT assay showed the cell viability of TamR MCF-7 cells transfected with siRNAs against EZH2 or control siRNAs for 72 h. Cells were treated with different concentrations of 4-OH TAM $(0-16 \mu \mathrm{M})$ for $48 \mathrm{~h}(\mathrm{n}=3)$. (F) MTT assay showed the cell viability of MCF-7 TamR cells transfected with siRNAs against EZH2 or control siRNAs for $72 \mathrm{~h}$. Cells were treated with $4-\mathrm{OH}$ TAM $(8 \mu \mathrm{M})$ for different times $0-72 \mathrm{~h}(\mathrm{n}=3)$. ${ }^{*} \mathrm{P}<0.05$ and ${ }^{* *} \mathrm{P}<0.01$ vs. control siRNAs. EZH2, enhancer of zeste homologue 2; 4-OH TAM, 4-hydroxytamoxifen; TamR, tamoxifen resistant; siRNA, small interfering RNA.

of cells in the G0/G1 phase was significantly decreased in EZH2-overexpressing MCF-7 cells compared with control cells $(\mathrm{P}<0.01$; Fig. $3 \mathrm{C}$ and $\mathrm{D})$. These results further suggested that EZH2 overexpression promoted cell cycle progression in MCF-7 cells.

Since cell cycle progression is promoted by cyclin-dependent kinases (CDKs) such as cyclin D1 and inhibited by CDK inhibitor p16, we then investigated the role of EZH2 in the expression of cyclin D1 and p16 in MCF-7 TamR cells. Western blot analysis showed that EZH2 knockdown significantly reduced cyclin D1 expression and increased p16 expression in MCF-7 TamR cells ( $\mathrm{P}<0.01$; Fig. 3E and F). In contrast, EZH2 overexpression increased cyclin D1 expression and decreased p16 expression in MCF-7 parental cells $(\mathrm{P}<0.01$; Fig. $3 \mathrm{E}$ and $\mathrm{F})$.

EZH2 knockdown decreases p16 gene methylation in MCF-7 TamR cells. We further investigated the role of EZH2 in p16 methylation in MCF-7 TamR cells and their parental cells, using methylation-specific PCR. As shown in Fig. 4A, EZH2 knockdown significantly decreased the DNA methylation level, but increased the unmethylation level of p16 in MCF-7 TamR cells $(\mathrm{P}<0.05$; Fig. 4A). In contrast, EZH2 overexpression significantly increased the DNA methylation level, but decreased unmethylation level of p16 in MCF-7 cells $(\mathrm{P}<0.05$; Fig. 4B).

The AKT and ERK signaling pathways are involved in EZH2 expression in MCF-7 TamR cells. It has been reported that AKT and ERK signaling pathway regulates EZH2 expression in breast cancer $(12,23,24)$. We then examined the expression of phosphorylated AKT, AKT, phosphorylated ERK1/2 and ERK1/2 in MCF-7 parental and TamR cells. Western blot analysis showed that the expression level of phosphorylated AKT, AKT, phosphorylated ERK1/2 and ERK1/2 was significantly lower in MCF-7 TamR cells compared with MCF-7 parental cells $(\mathrm{P}<0.01$; Fig. $5 \mathrm{~A}$ and $\mathrm{B})$. These results suggested that the AKT and ERK signaling pathways are involved in EZH2 expression in MCF-7 TamR cells.

\section{Discussion}

In the present study, we examined EZH2 expression in MCF-7 TamR cells, and found that EZH2 expression was significantly increased in MCF-7 TamR cells compared with parental control cells. We further found that EZH2 overexpression decreased the sensitivity of MCF-7 cells to tamoxifen, and 

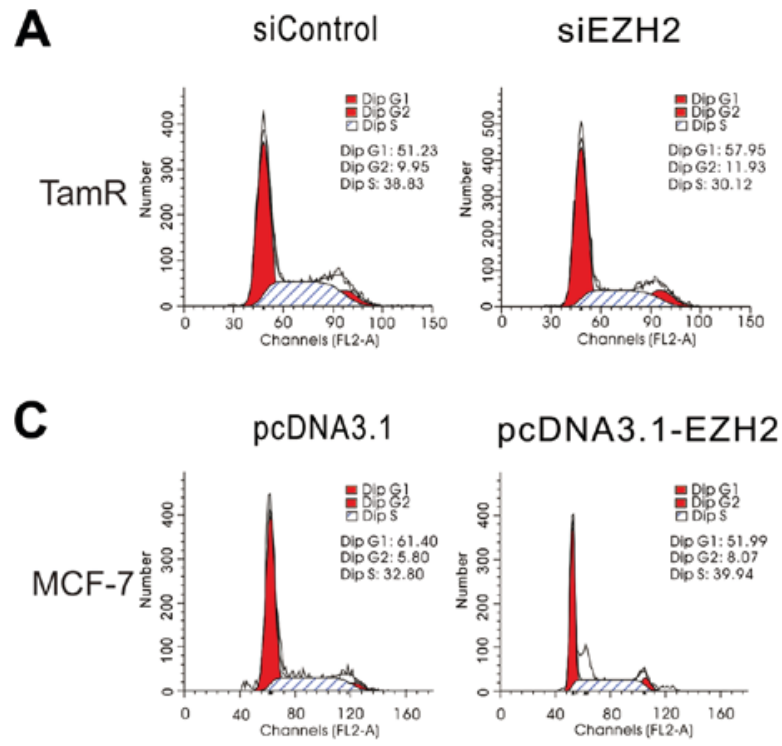

E

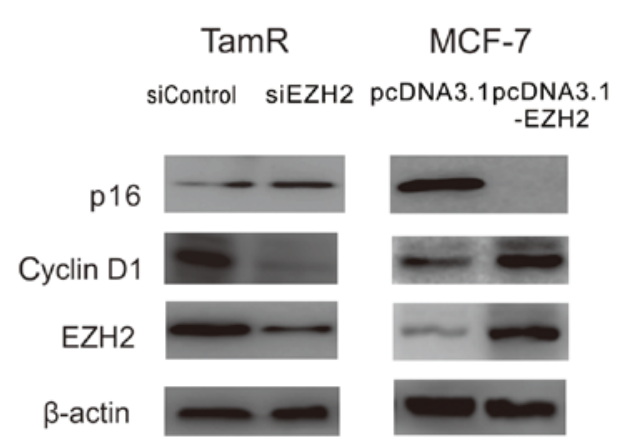

B

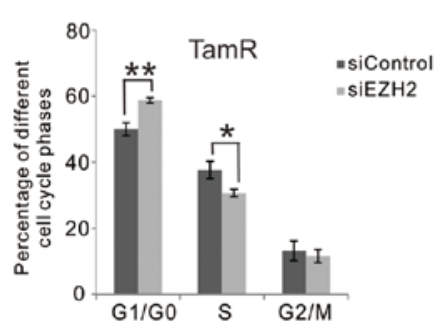

D

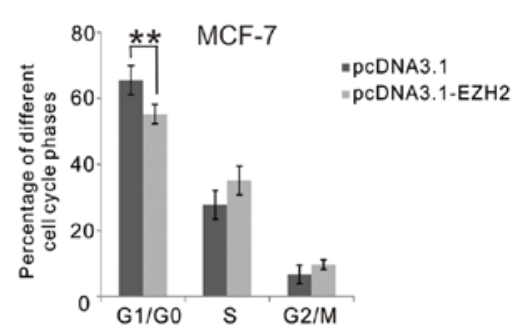

$\mathbf{F}$
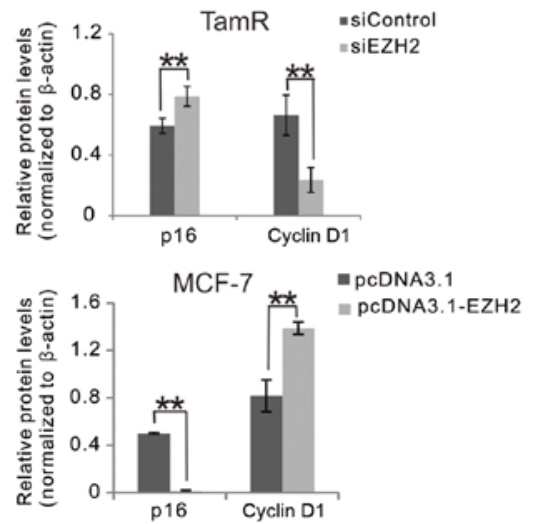

Figure 3. EZH2 affected the cell cycle in MCF-7 TamR and their parental cells. (A) Representative flow cytometry of the cell-cycle distribution in MCF-7 TamR cells transfected with siRNAs against EZH2 or control siRNAs for $72 \mathrm{~h}$. Cells were treated with $8 \mu \mathrm{M} 4-\mathrm{OH}$ TAM for $48 \mathrm{~h}$. (B) The percentages of MCF-7 TamR cells transfected with siRNAs against EZH2 or control siRNAs in the G0/G1, S and G2/M phases (n=3). (C) Representative flow cytometry depicting the cell-cycle distribution in MCF-7 cells transfected with pcDNA3.1-EZH2 expression vector or pcDNA3.1 vector for $72 \mathrm{~h}$. Cells were treated with $8 \mu \mathrm{M}$ 4-OH TAM for $48 \mathrm{~h}$. (D) The percentage of MCF-7 cells transfected with pcDNA3.1-EZH2 expression vector or pcDNA3.1 vector in the G0/G1, S and G2/M phases (n=3). (E) Representative western blotting of the expression of p16, cyclin D1 and EZH2 in MCF-7 TamR cells transfected with siRNAs against EZH2 or control siRNAs for $72 \mathrm{~h}$, or in MCF-7 cells transfected with pcDNA3.1-EZH2 expression vectors or pcDNA3.1 empty vectors for $72 \mathrm{~h}$. Cells were treated with $8 \mu \mathrm{M}$ 4-OH TAM for $48 \mathrm{~h}$. $\beta$-actin was used as a loading control. (F) Quantification of the expression of cycle D1 and p16 (n=3). "P<0.05 and ${ }^{* *} \mathrm{P}<0.01$, as indicated. EZH2, enhancer of zeste homologue 2; 4-OH TAM, 4-hydroxytamoxifen; TamR, tamoxifen resistant; siRNA, small interfering RNA.

EZH2 knockdown increased the sensitivity of MCF-7 TamR cells. Furthermore, we found that EZH2 knockdown induced cell cycle arrest in MCF-7 TamR cells by decreasing Cyclin D1 expression and increasing p16 expression. Moreover, EZH2 knockdown reduced p16 gene methylation in MCF-7 TamR cells. Our findings suggest that EZH2 overexpression contributes to tamoxifen resistance in breast cancer, and EZH2 inhibition may reverse tamoxifen resistance in breast cancer by regulating cell cycle via the demethylation of the p16 gene.

EZH2 overexpression has been found in many tumors including breast cancer (12). However, the mechanisms underlying EZH2 overexpression in breast cancer are not fully understood. It has been reported that the AKT and MEK/ERK signaling pathways promoted EZH2 overexpression in breast cancer $(12,23,24)$. In the present study, we found that EZH2 was overexpressed in MCF-7 TamR cells. However, the mechanisms underlying EZH2 overexpression in TamR cells remain unclear. It has been known that AKT and MEK/ERK signaling activation contributes to tamoxifen resistance in breast cancer $(25,26)$. We found that the expression of AKT, p-AKT, ERK1/2, p-ERK1/2 significantly reduced in MCF-7 TamR cells than their parental cells. Therefore, it appears that AKT and MEK/ERK signaling may be responsible for EZH2 overexpression in TamR cells. Further studies are warranted to investigate the signaling mechanisms underlying EZH2 overexpression in TamR breast cancer cells.

Cell cycle regulators such as cyclins, CDKs and CDK inhibitors play an important role in regulation of cell cycle progression and have been demonstrated to be associated with tamoxifen resistance (26). Cyclin D1 promotes cell cycle progression to the $\mathrm{S}$ phase, and has been found to be upregulated in tamoxifen-resistant breast cancer cells (27). Cyclin D1 overexpression is associated with poor clinical outcomes in breast cancer patients following tamoxifen 
A
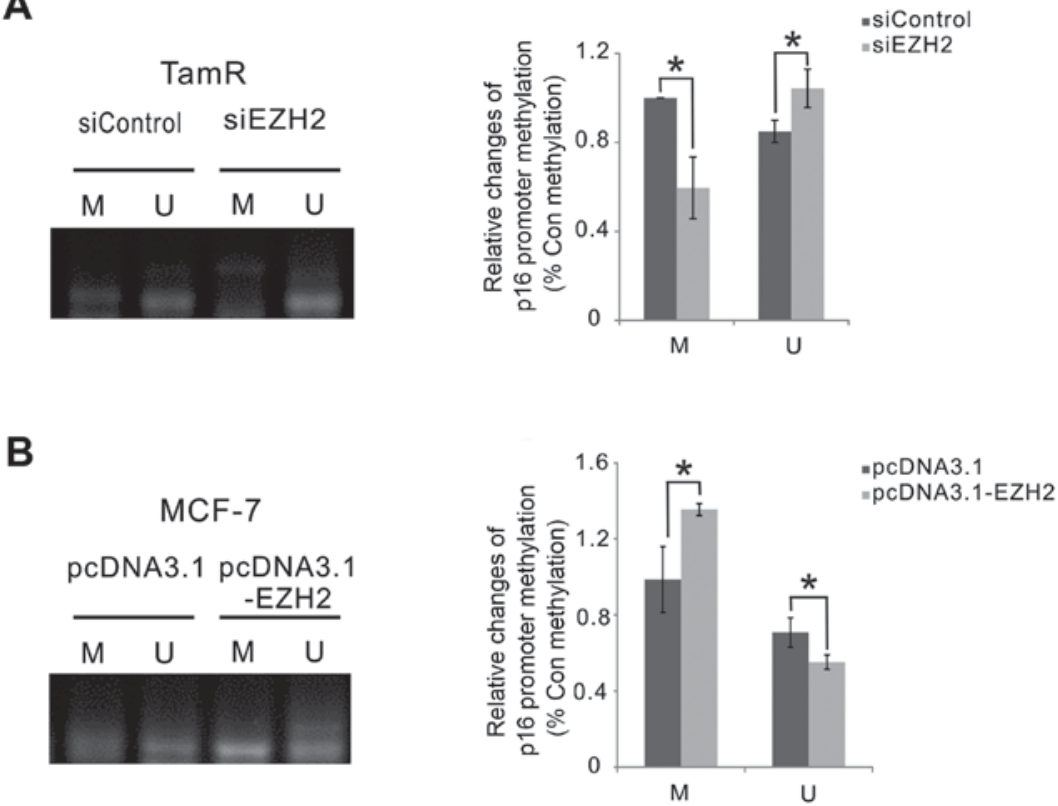

Figure 4. EZH2 affected the methylation of p16 in MCF-7 TamR and their parental cells. (A) MCF-7 TamR cells were transfected with siRNAs against EZH2 or control siRNAs for $72 \mathrm{~h}$, and were then treated with $8 \mu \mathrm{M} 4-\mathrm{OH}$ TAM for $48 \mathrm{~h}$. MSP analysis was performed to detect the p16 promoter methylation. (B) MCF-7 cells were transfected with pcDNA3.1-EZH2 expression vector or pcDNA3.1 vector for $72 \mathrm{~h}$, and were then treated with $8 \mu \mathrm{M} 4-\mathrm{OH}$ TAM for $48 \mathrm{~h}$. MSP analysis was performed to detect the p16 promoter methylation $(\mathrm{n}=3)$. " $\mathrm{P}<0.05$, as indicated. EZH2, enhancer of zeste homologue 2; 4-OH TAM, 4-hydroxytamoxifen; TamR, tamoxifen resistant; siRNA, small interfering RNA; MSP, methylation-specific polymerase chain reaction; U, unmethylated; M, methylated.

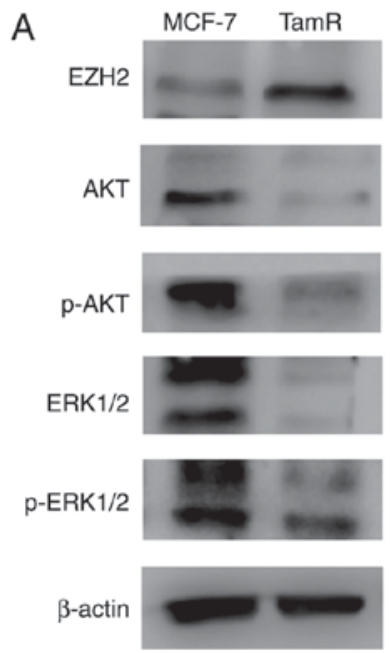

$\mathrm{B}$

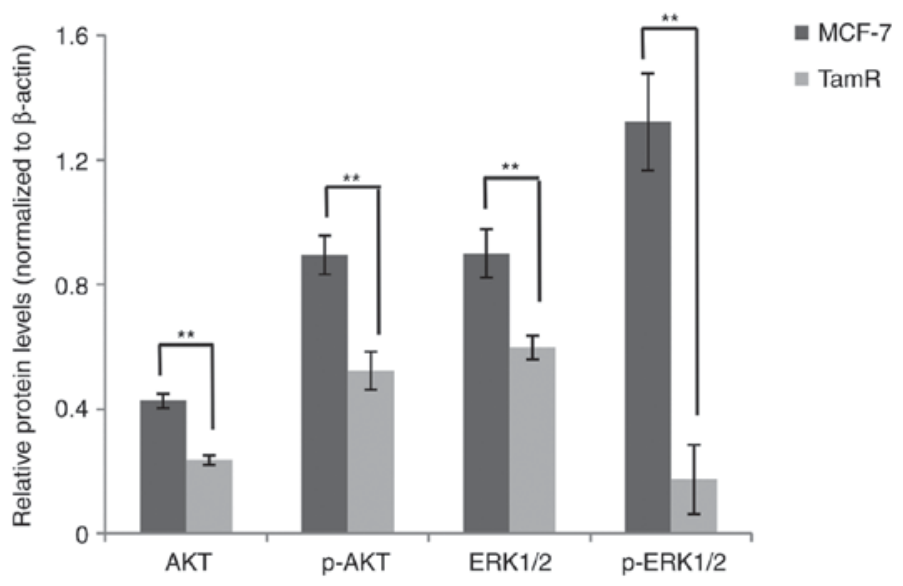

Figure 5. The expression of p-AKT, AKT, p-ERK1/2 and ERK1/2 in MCF-7 parental and TamR cells. (A) Western blot analysis revealing the protein expression of EZH2, AKT, p-AKT, ERK1/2 and p-ERK1/2 in MCF-7 TamR and their parental cells. $\beta$-actin was used as the loading control. (B) Quantification of AKT, p-AKT, ERK1/2 and p-ERK1/2 expression $(n=3) . ~{ }^{* *} \mathrm{P}<0.01$, as indicated. EZH2, enhancer of zeste homologue 2; TamR, tamoxifen resistant; $\mathrm{p}-$, phosphorylated; AKT, protein kinase B; ERK, extracellular signal-regulated kinase.

treatment $(28,29)$. It has been reported that cyclin D1 expression is necessary for proliferation of tamoxifen-resistant breast cancer cells (30). In the present study, we found that EZH2 knockdown increased the sensitivity of MCF-7 TamR cells to tamoxifen, and induced cell cycle arrest in MCF-7 TamR cells by inhibiting cyclin D1 expression. In addition, EZH2 overexpression increased cyclin D1 expression in MCF-7 parental cells, accompanied by a decrease in tamoxifen sensitivity. These findings suggest that EZH2 overexpression contributes to tamoxifen resistance in breast cancer cells by upregulating cyclin D1.
As a tumor suppressor, p16 is a cyclin-dependent kinase inhibitor that binds to CDK4/6, and subsequently prevents the interaction of CDK4/6 with cyclin D1, resulting in inhibition of cell cycle progression (31). In this study, we found that EZH2 knockdown induced cell cycle arrest and increased p16 expression in MCF-7 TamR cells, and EZH2 overexpression decreased p16 expression in MCF-7 parental cells, suggesting that EZH2 knockdown inhibits cell cycle progression by upregulation of p16. Hypermethylation of the p16 gene is one of the major mechanisms responsible for downregulation of p16 expression in many cancers including breast cancer, leading 
to cell cycle progression (32-34). In this study, we found that EZH2 knockdown reduced the DNA methylation level of the p16 promoter in MCF-7 TamR cells, suggesting that EZH2 knockdown upregulates p16 expression via inhibition of p16 promoter methylation. It has been reported that EZH 2 controls DNA methylation by directly interacting with the DNA methyltransferases (35). Therefore, EZH2 may promote p16 promoter methylation via DNA methyltransferase.

It has been reported that an increase in ER expression can re-sensitize TamR cells to tamoxifen in breast cancer cells (36), suggesting that downregulation of ER expression may contribute to tamoxifen resistance. In the present study, we examined ER expression in MCF-7 parental and TamR cells, and found that ER expression was not significantly different between MCF-7 parental and TamR cells, suggesting that tamoxifen resistance might be mediated by ER-independent signaling pathways in MCF-7 TamR cells.

Several studies have shown that EZH2 overexpression correlates with pathological types, histological grade, ER negativity, PR negativity, and HER-2 positivity as well as poor prognosis in breast cancer $(17,37,38)$. In addition, low EZH2 expression is associated with a reduced risk of developing breast cancer (39). It has been reported that EZH2 inhibition decreases proliferation and promotes apoptosis in breast cancer cells in vitro, and produces anti-tumor activity in vivo $(40,41)$. Therefore, EZH2 inhibition may represent a promising new therapeutic strategy for the treatment of breast cancer. Several small molecule inhibitors of methyl transferase have been identified for the treatment of many cancers such as breast cancer and leukemia, and combination therapy with hypomethylators and chemotherapeutic agents can synergistically inhibit breast cancer cells (42-44). Recently, Song et al (40) reported that ZLD1039, a highly selective small molecule inhibitor of EZH2, inhibited tumor growth and metastasis in breast cancer xenograft mice. Furthermore, a phase I/II clinical trial has been initiated for EZH2 inhibitor EPZ-6438 for the treatment of advanced solid tumor (45). In this study, we found that EZH2 inhibition increased the sensitivity of MCF-7 TamR breast cancer cells to tamoxifen, suggesting that EZH2 inhibitors may be used to reverse tamoxifen resistance, and combination of EZH2 inhibitors with tamoxifen may be effective for treating ER-positive breast cancer.

In summary, we found that EZH2 was overexpressed in MCF-7 TamR breast cancer cells, and EZH2 knockdown by siRNAs increased the sensitivity of MCF-7 TamR cells to tamoxifen. In addition, EZH2 knockdown induced cell cycle arrest by decreasing cyclin D1 expression and increasing p16 expression. Inhibition of p16 promoter methylation by EZH2 knockdown resulted in upregulation of p16 expression. Our findings suggest that EZH2 inhibition may be used for reversing tamoxifen resistance in breast cancer.

\section{Acknowledgements}

The present study was supported by grants from the Natural Science Foundations of Liaoning Province of China (no. 2015020723) to Yue Fang, and (no. 2015020501) to Fan Yao. This study was also supported by Research Fund for the Doctoral Program of Higher Education of Liaoning Province, China (no. 20091110) to Yue Fang.

\section{References}

1. Global Burden of Disease Cancer Collaboration; Fitzmaurice C, Dicker D, Pain A, Hamavid H, Moradi-Lakeh M, MacIntyre MF, Allen C, Hansen G, Woodbrook R, et al: The Global burden of cancer 2013. JAMA Oncol 1: 505-527, 2015.

2. Li F, Dou J, Wei L, Li S and Liu J: The selective estrogen receptor modulators in breast cancer prevention. Cancer Chemother Pharmacol 77: 895-903, 2016.

3. Rondón-Lagos M, Villegas VE, Rangel N, Sanchez MC and Zaphiropoulos PG: Tamoxifen resistance: Emerging molecular targets. Int J Mol Sci 17: E1357, 2016.

4. Wrobel K, Zhao YC, Kulkoyluoglu E, Chen KL, Hieronymi K, Holloway J, Li S, Ray T, Ray PS, Landesman Y, et al: ER $\alpha-X P O 1$ cross talk controls tamoxifen sensitivity in tumors by altering ERK5 cellular localization. Mol Endocrinol 30: 1029-1045, 2016.

5. Luqmani YA and Alam-Eldin N: Overcoming resistance to endocrine therapy in breast cancer: New approaches to a nagging problem. Med Princ Pract 25 (Suppl 2): S28-S40, 2016.

6. Mahara S, Lee PL, Feng M, Tergaonkar V, Chng WJ and Yu Q: HIFI- $\alpha$ activation underlies a functional switch in the paradoxical role of Ezh2/PRC2 in breast cancer. Proc Natl Acad Sci USA 113: E3735-E3744, 2016.

7. Shenoy N, Vallumsetla N, Zou Y, Galeas JN, Shrivastava M, Hu C, Susztak K and Verma A: Role of DNA methylation in renal cell carcinoma. J Hematol Oncol 8: 88, 2015.

8. Qi W, Chan H, Teng L, Li L, Chuai S, Zhang R, Zeng J, Li M, Fan H, Lin Y, et al: Selective inhibition of Ezh2 by a small molecule inhibitor blocks tumor cells proliferation. Proc Natl Acad Sci USA 109: 21360-21365, 2012.

9. Hubaux R, Thu KL, Coe BP, MacAulay C, Lam S and Lam WL: EZH2 promotes E2F-driven SCLC tumorigenesis through modulation of apoptosis and cell-cycle regulation. J Thorac Oncol 8: 1102-1106, 2013.

10. Cai L, Wang Z and Liu D: Interference with endogenous EZH2 reverses the chemotherapy drug resistance in cervical cancer cells partly by up-regulating dicer expression. Tumour Biol 37: 6359-6369, 2016.

11. Sun R, Shen J, Gao Y, Zhou Y, Yu Z, Hornicek F, Kan Q and Duan Z: Overexpression of EZH2 is associated with the poor prognosis in osteosarcoma and function analysis indicates a therapeutic potential. Oncotarget 7: 38333-38346, 2016.

12. Fujii S, Tokita K, Wada N, Ito K, Yamauchi C, Ito Y and Ochiai A: MEK-ERK pathway regulates EZH2 overexpression in association with aggressive breast cancer subtypes. Oncogene 30: 4118-4128, 2011.

13. Varambally S, Dhanasekaran SM, Zhou M, Barrette TR, Kumar-Sinha C, Sanda MG, Ghosh D, Pienta KJ, Sewalt RG, Otte AP, et al: The polycomb group protein EZH2 is involved in progression of prostate cancer. Nature 419: 624-629, 2002.

14. Kim KH and Roberts CW: Targeting EZH2 in cancer. Nat Med 22: 128-134, 2016.

15. Wassef M, Michaud A and Margueron R: Association between EZH2 expression, silencing of tumor suppressors and disease outcome in solid tumors. Cell Cycle 15: 2256-2262, 2016.

16. Italiano A: Role of the EZH2 histone methyltransferase as a therapeutic target in cancer. Pharmacol Ther 165: 26-31, 2016.

17. Jang SH, Lee JE, Oh MH, Lee JH, Cho HD, Kim KJ, Kim SY, Han SW, Kim HJ, Bae SB and Lee HJ: High EZH2 protein expression is associated with poor overall survival in patients with luminal a breast cancer. J Breast Cancer 19: 53-60, 2016.

18. Reijm EA, Timmermans AM, Look MP, Meijer-van Gelder ME, Stobbe CK, van Deurzen CH, Martens JW, Sleijfer S, Foekens JA, Berns PM and Jansen MP: High protein expression of EZH2 is related to unfavorable outcome to tamoxifen in metastatic breast cancer. Ann Oncol 25: 2185-2190, 2014

19. Knowlden JM, Hutcheson IR, Jones HE, Madden T, Gee JM, Harper ME, Barrow D, Wakeling AE and Nicholson RI: Elevated levels of epidermal growth factor receptor/c-erbB2 heterodimers mediate an autocrine growth regulatory pathway in tamoxifen-resistant MCF-7 cells. Endocrinology 144: 1032-1044, 2003.

20. Coward WR, Feghali-Bostwick CA, Jenkins G, Knox AJ and Pang L: A central role for G9a and EZH2 in the epigenetic silencing of cyclooxygenase- 2 in idiopathic pulmonary fibrosis. FASEB J 28: 3183-3196, 2014.

21. Velagapudi SP, Gallo SM and Disney MD: Sequence-based design of bioactive small molecules that target precursor microRNAs. Nat Chem Biol 10: 291-297, 2014. 
22. Pan FP, Zhou HK, Bu HQ, Chen ZQ, Zhang H, Xu LP, Tang J, Yu QJ, Chu YQ, Pan J, et al: Emodin enhances the demethylation by 5-Aza-CdR of pancreatic cancer cell tumor-suppressor genes P16, RASSF1A and ppENK. Oncol Rep 35: 1941-1949, 2016.

23. Chang LC, Lin HY, Tsai MT, Chou RH, Lee FY, Teng CM Hsieh MT, Hung HY, Huang LJ, Yu YL and Kuo SC: YC-1 inhibits proliferation of breast cancer cells by down-regulating EZH2 expression via activation of $\mathrm{c}-\mathrm{Cbl}$ and ERK. Br J Pharmacol 171: 4010-4025, 2014.

24. Cha TL, Zhou BP, Xia W, Wu Y, Yang CC, Chen CT, Ping B, Otte AP and Hung MC: Akt-mediated phosphorylation of EZH2 suppresses methylation of lysine 27 in histone $\mathrm{H} 3$. Science 310 306-310, 2005.

25. Riggins RB, Schrecengost RS, Guerrero MS and Bouton AH: Pathways to tamoxifen resistance. Cancer Lett 256: 1-24, 2007.

26. Viedma-Rodriguez R, Baiza-Gutman L, Salamanca-Gómez F, Diaz-Zaragoza M, Martínez-Hernández G, Ruiz Esparza-Garrido R, Velázquez-Flores MA and Arenas-Aranda D: Mechanisms associated with resistance to tamoxifen in estrogen receptor-positive breast cancer (Review). Oncol Rep 32: 3-15, 2014.

27. Kilker RL, Hartl MW, Rutherford TM and Planas-Silva MD: Cyclin D1 expression is dependent on estrogen receptor function in tamoxifen-resistant breast cancer cells. J Steroid Biochem Mol Biol 92: 63-71, 2004.

28. Stendahl M, Kronblad A, Rydén L, Emdin S, Bengtsson NO and Landberg G: Cyclin D1 overexpression is a negative predictive factor for tamoxifen response in postmenopausal breast cancer patients. Br J Cancer 90: 1942-1948, 2004.

29. Rudas M, Lehnert M, Huynh A, Jakesz R, Singer C, Lax S, Schippinger W, Dietze O, Greil R, Stiglbauer W, et al: Cyclin D1 expression in breast cancer patients receiving adjuvant tamoxifen-based therapy. Clin Cancer Res 14: 1767-1774, 2008.

30. Kilker RL and Planas-Silva MD: Cyclin D1 is necessary for tamoxifen-induced cell cycle progression in human breast cancer cells. Cancer Res 66: 11478-11484, 2006.

31. Hara E, Smith R, Parry D, Tahara H, Stone S and Peters G: Regulation of p16CDKN2 expression and its implications for cell immortalization and senescence. Mol Cell Biol 16: 859-867, 1996.

32. Shim YH, Park HJ, Choi MS, Kim JS, Kim H, Kim JJ, Jang JJ and Yu E: Hypermethylation of the p16 gene and lack of p16 expression in hepatoblastoma. Mod Pathol 16: 430-436, 2003

33. Lee JJ, Ko E, Cho J, Park HY, Lee JE, Nam SJ, Kim DH and Cho EY: Methylation and immunoexpression of p16 (INK4a) tumor suppressor gene in primary breast cancer tissue and their quantitative p16 (INK4a) hypermethylation in plasma by real-time PCR. Korean J Pathol 46: 554-561, 2012.
34. Wang L, Tang L, Xie R, Nie W, Chen L and Guan X: p16 promoter hypermethylation is associated with increased breast cancer risk. Mol Med Rep 6: 904-908, 2012.

35. Vire E, Brenner C, Deplus R, Blanchon L, Fraga M, Didelot C, Morey L, Van Eynde A, Bernard D, Vanderwinden JM, et al: The Polycomb group protein EZH2 directly controls DNA methylation. Nature 439: 871-874, 2006.

36. Li Y, Meeran SM and Tollefsbol TO: Combinatorial bioactive botanicals re-sensitize tamoxifen treatment in ER-negative breast cancer via epigenetic reactivation of ER $\alpha$ expression. Sci Rep 7: 9345, 2017.

37. Wang X, Hu B, Shen H, Zhou H, Xue X, Chen Y, Chen S, Han Y, Yuan B, Zhao H, et al: Clinical and prognostic relevance of EZH2 in breast cancer: A meta-analysis. Biomed Pharmacother 75: 218-225, 2015.

38. Guo S, Li X, Rohr J, Wang Y, Ma S, Chen P and Wang Z: EZH2 overexpression in different immunophenotypes of breast carcinoma and association with clinicopathologic features. Diagn Pathol 11: 41, 2016

39. Beca F, Kensler K, Glass B, Schnitt SJ, Tamimi RM and Beck AH: EZH2 protein expression in normal breast epithelium and risk of breast cancer: Results from the nurses' health studies. Breast Cancer Res 19: 21, 2017.

40. Song X, Gao T, Wang N, Feng Q, You X, Ye T, Lei Q, Zhu Y, Xiong M, Xia Y, et al: Selective inhibition of EZH2 by ZLD1039 blocks H3K27 methylation and leads to potent anti-tumor activity in breast cancer. Sci Rep 6: 20864, 2016.

41. Zhang L, Deng L, Chen F, Yao Y, Wu B, Wei L, Mo Q and Song Y: Inhibition of histone H3K79 methylation selectively inhibits proliferation, self-renewal and metastatic potential of breast cancer. Oncotarget 5: 10665-10677, 2014.

42. Feng Z, Yao Y, Zhou C, Chen F, Wu F, Wei L, Liu W, Dong S, Redell M, Mo Q and Song Y: Pharmacological inhibition of LSD1 for the treatment of MLL-rearranged leukemia. J Hematol Oncol 9: 24, 2016.

43. Song Y, Wu F and Wu J: Targeting histone methylation for cancer therapy: Enzymes, inhibitors, biological activity and perspectives. J Hematol Oncol 9: 49, 2016.

44. Cang S, Ma Y, Chiao JW and Liu D: Phenethyl isothiocyanate and paclitaxel synergistically enhanced apoptosis and alpha-tubulin hyperacetylation in breast cancer cells. Exp Hematol Oncol 3: 5, 2014.

45. Huang T, Lin C, Zhong LL, Zhao L, Zhang G, Lu A, Wu J and Bian Z: Targeting histone methylation for colorectal cancer. Therap Adv Gastroenterol 10: 114-131, 2017. 